\title{
AMBULATÓRIO DE SEXOLOGIA DO INSTITUTO DE GINECOLOGIA DA UFRJ \\ - HOSPITAL MONCORVO FILHO
}

Maria do Carmo de Andrade e Silva ; Paulo Roberto Bastos Canella ${ }^{2}$

\author{
THE AMBULATORY OF SEXOLOGY \\ OF GYNECOLOGY INSTITUTE OF UFRJ
}

Resumo: O Ambulatório de Sexologia do Instituto de Ginecologia da UFRJ. - Hospital Moncorvo Filho foi organizado em 1986 e desde então se encontra em funcionamento. Neste serviço médicos e psicólogos trabalham em equipe, em um processo multidisciplinar para o atendimento dos diversos problemas sexuais. A população que freqüenta este ambulatório caracteriza-se, por pessoas residentes na periferia da cidade do Rio de Janeiro e, que têm carências socioeconômicas e culturais acentuadas. A demanda de queixas apresentadas é muito variada, trabalhando-se com disfunçōes sexuais femininas e masculinas, conflitos e inversōes de identidade, intersexo, má formaçōes genitais, transtôrno de gênero e conflitos conjugais. Problemas com etiologias orgânicas, psicológicas, sociais e mistas. A partir do inicio do trabalho terapêutico, a pessoa será atendida sempre pelo mesmo profissional, ou por profissionais, quando o caso exigir uma participação médico-psicológica mais constante. Quando a pessoa tem um parceiro fixo - e para um melhor andamento do caso, a presença dele for importante, este será chamado a participar. Neste caso poder-se disponibilizar atendimentos individuais e atendimentos mistos, às pessoas e ao casal. Nestes anos de trabalho em equipe multidisplinar, temos observado condições de trabalho mais seguras, assim como melhores resultados com esta população. Que apresentando problemas variados, muitas vezes implicando causas mistas, requerem atendimentos com focos mais amplos.

Psicóloga. Mestre em Psicologia e Livre Docente em Sexualidade Humana. Coordenadora do Ambulatório de Sexologia do Instituto de Ginecologia da UFRJ.e-mail: mariacarmoas@yahoo. com.br

2 Médico Ginecologista - Mestre em Ginecologia e Livre Docente em Medicina.Coordenador do Ambulatório de Sexologia do Instituto de Ginecologia da UFRJ. e-mail: canella@gineco.ufrj.br 
Palavras-chave: Atendimento ambulatorial; problemas sexuais; sexologia clínica; atendimento multidisciplinar.

\begin{abstract}
The Ambulatory of Sexology of Gynecology Institute of UFRJ Hospital Moncorvo Filho was created in 1986 and is in continuous operation since then. The service is an available to patients with several sexual problems and has a trained doctors and psychologists who worker as a team in a multidisciplinary process. The patients come from poor Rio de Janeiro areas, having deficient social, economic and cultural conditions. The main complains are male and female sexual dysfunctions, identity inversion and conflict, genital malformation, gender disorders, and couple conflict. In summary problems are organic, psychological, social and frequently a combination of two or more factors. Thought out the therapeutic work the patient is assisted for the same professional or for the same team of professionals, when a coordinated doctor - psychologist work is required. When the patient has a regular partner he will normally be invited to participate in the treatment. In this case individual and couple sessions are made available to produce better and faster results. The coordination group in all those years of work has noticed that the multidiscipline team obtained improved working conditions and better results them the traditional one only professional assistance. Most problems showed mixed causes and symptoms, requiring wide focus.
\end{abstract}

Keywords: Ambulatory assistance; sexual problems; clinical sexology; multidisciplinary assistance.

Os atendimentos ambulatoriais na área de sexologia no Brasil, ainda são incomuns e pouco estruturados. O Ambulatório de Sexologia do Instituto de Ginecologia da UFRJ. - Hospital Moncorvo Filho foi organizado em 1986 pelo Prof. Dr. Paulo Roberto Bastos Canella (Médico Ginecologista) e pelo Prof. Dr. Araguari Chalar da Silva (Psicólogo). Tal iniciativa pioneira sofreu algumas interrupçōes nos anos iniciais de sua implantação. No entanto, apesar das várias dificuldades por que tem passado, continua resistido às dificuldades e atualmente encontra-se sob a coordenação do Dr. Paulo Canella e Dra.Maria do Carmo de Andrade Silva (Psicóloga). Profissionais que a muito desenvolvem trabalho em sexologia clínica, tendo como base um procedimento multidisciplinar de estudo, pesquisa e atuação conjunta.

Compondo também o quadro regular de profissionais deste serviço atualmente encontram-se: Dra.Maria Luiza Mecedo Araújo (Psicóloga), Rosimeri Eirado 
Bruno (Ginecologista e Mestre em Sexologia), Alfredo Donis Romero (Médico, Cirurgiāo Vascular e Mestre em Sexologia) e Silvia V. Machado Teixeira (Psicóloga e Mestre em Sexologia).

A população que freqüenta este ambulatório caracteriza-se em sua maioria, por mulheres residentes na periferia da cidade do Rio de Janeiro e, que têm carências socioeconômicas e culturais acentuadas. Portanto pouquíssimo acesso a esclarecimentos sexuais. As interaçōes familiares em que vivem, estruturamse baseadas em casamentos típicos, onde os homens ainda são a representação do poder e a sexualidade deles a que mais importa, ficando a mulher com os papeis mais tradicionais de esposa, dona de casa e mãe.

Mesmo com todos estes limites, as notícias de possibilidades de realização sexual feminina, gradativamente têm atingido também a esta parcela da população. E as queixas de dificuldades sexuais apresentam-se nas consultas ginecológicas, sempre que algum espaço é percebido. Pois para estas mulheres, muitas vezes a consulta ao ginecologista é o único espaço oficial confiável, em que por vezes, conseguem falar e obter algum esclarecimento mais específico, sobre a temática sexual e suas dificuldades pessoais.

Porém problemas sexuais são questões amplas e muitas vezes os ginecologistas, não dispõe de espaço de tempo, local adequado ou mesmo formação específica, para o trabalho com dificuldades sexuais em ambulatórios públicos. Locais onde o tempo já é escasso, até mesmo para as demandas essencialmente ginecológicas. Assim premidos pelas diversas queixas sexuais e impossibilitados de prestar auxílio mais adequado, a criação de um ambulatório de Sexologia no Instituto de Ginecologia, foi a solução encontrada.

As clientes que chegavam ao ambulatório de sexologia, inicialmente nos eram encaminhadas exclusivamente dos outros ambulatórios do Hospital, no entanto logo observamos a necessidade de atendimento, por vezes também aos parceiros destas mulheres. E com o passar dos anos temos recebido encaminhamentos de outros hospitais da rede pública, que não dispõe deste tipo de serviço e, que nos remetem pacientes, o que nos causa grande demanda, muitas vezes difícil de administrar.

Quando a mulher vem encaminhada do próprio hospital, ao marcar sua consulta inicial no ambulatório de sexologia, seu prontuário ginecológico já a acompanha, favorecendo que tenhamos uma idéia de sua história clínica ginecológica, processo que pode nos auxiliar em determinados casos, na constituição de um diagnóstico diferencial. Porém, quando a (o) paciente advém de outra instituição, uma avaliação médica torna-se necessária, antes de prosseguirmos o trabalho com a queixa sexual. 
Feita a triagem inicial, posterior diagnóstico da problemática apresentada e, confirmando o foco em uma dificuldade sexual, a pessoa passa a ser atendida regularmente no ambulatório, uma vez por semana ou de 15 em 15 dias. Tal periodicidade dependerá muitas vezes das possibilidades dos clientes, até mais do que do ideal de acompanhamento do caso, pois como já mencionamos, trabalhamos com uma parcela da população claramente desfavorecida, que reside em locais distantes do Hospital, que trabalha e tem família extensa, apresentando reais dificuldades de comparecer com a freqüência desejada.

A partir do inicio do trabalho terapêutico, a pessoa será atendida sempre pelo mesmo profissional, ou por profissionais quando o caso exigir uma participação médico-psicológica constante.

Quando a pessoa tem um parceiro fixo - e para um melhor andamento do caso, a presença dele for importante, este será chamado a participar. Neste caso pode-se disponibilizar atendimentos individuais e atendimentos mistos (às pessoas e ao casal). Em vários casos observamos, a necessidade de algum mínimo trabalho com a unidade conjugal, para que qualquer alteração possa se efetivar, no que se refere à unidade sexual.

Para o atendimento neste ambulatório dispomos de pequenos boxes para as entrevistas, com pouca privacidade, uma sala de exame médico privativa, que é utilizada também para exercícios de relaxamento, sensibilização, recursos áudio visual, exames e auto exames e uma pequena sala para pequenos grupos.

Como metodologia teórica, baseamo-nos principalmente nos tabalhos de Masters e Johnson (1966, 1970, 1997), Kaplan (1977, 1983, 1983, 1999), Lazarus (1977, 1977), Heiman e Lopiccolo (1981), Barbach (1975), Zilbergeld (1978), Fiorini, (1977, 1978), Papp (2002), Canella e AndradeSilva (2003), Andrade-Silva, Canella, Romero e Burno (2005), adaptando-os à realidade de nossa cultura e especialmente à esta população e as limitações deste ambulatório.

Utilizamo-nos de triagem, anamnese estruturada, entrevistas, exames físicos, laboratoriais e específicos, medicações, esclarecimentos com auxílio de material gráfico ou áudio visual, auto exames, desenvolvimento de permissão, revivência de experiências traumáticas, relaxamento, dessenbilização e sensibilização, moldes dilatadores adaptados, grupo de reflexão e esclarecimento, atendimento ao casal, desenvolvimento de sensaçōes e percepçōes, exercícios de kegel, desenvolvimento de auto conceito, auto estima e auto imagem, desenvolvimento de comunicação interpessoal do casal, exercícios para serem realizados em casa (individuais ou para o casal). 
Neste serviço em 1999, por fazermos parte à época de um Curso de Mestrado em Sexologia, implantamos e implementamos um Projeto de Pesquisa, denominado Gênero e Transgenitalização. Esta iniciativa se deu, especialmente em função de que, neste ambulatório, já vivenciávamos na prática o atendimento a indivíduos, que apresentavam conflitos ou inversōes em suas identidades de gênero nos casos diagnosticados como intersexo, ou seja, indivíduos com má formação genital ou genitais ambíguos. Casos existentes neste Instituto em função de sua antiga tradição em ginecologia e reprodução. Assim, a partir desta base inicial e por já termos pessoas com queixas de Transtorno de gênero neste serviço, demos início a este novo Projeto, que envolveu outros profissionais e uma estrutura mais complexa de atendimentos.

Como já mencionado estamos localizados em um Instituto Universitário de Ginecologia, com isso as queixas mais freqüentemente apresentadas neste ambulatório, nos últimos 5 anos são: Disfunçōes Sexuais Femininas, perfazendo (84\%) de nossos atendimentos, Disfunçōes Sexuais Masculinas, (9.5\%), Transtorno de Gênero (2.5\%), Esclarecimentos e ou Encaminhamentos (1.5\%), Má Formaçōes Genitais (1.5\%), Conflitos Homosexuais (1.0\%), Travestismo $(0.25 \%)$.

Quanto às incidências de diagnósticos das principais disfunções sexuais femininas apresentas, temos a relatar: Anorgasmia Secundária (32\%), Inibição ou Diminuição de Desejo (29\%), Anorgasmia Primária (21\%), Vaginismo (13\%), Dispareunia (5\%). Porém observamos alta incidência de queixas associadas de Inibição de Desejo e Anorgasmia em mais de metade dos casos.

Pensamos importante referir que as queixas de Transtorno de Desejo, apresentam-se não só associadas a outras disfunções, como também a sérios conflitos conjugais. E por necessidade de manutenção do relacionamento, em função de problemas socioeconômicos que envolvem filhos e moradia, os desentendimentos conjugais, assim como o alcoolismo e a agressividade masculina, na perspectiva dessas mulheres, precisam ficar subjulgados, para que o relacionamento possa ter continuidade. Assim, muitas delas procuram atendimento para seu Transtorno de desejo, sem que tenham feito correlação entre tais circunstâncias vivenciais e seu não desejo sexual.

Por tudo isso, apesar do Ambulatório de Sexologia ter como objetivo principal os problemas sexuais, em vários casos, atendemos a casais. Casais em que a queixa sexual é claramente secundária, sendo os conflitos conjugais o mais significante naquela relação, levando a maiores dificuldades e complexidade de trabalho terapêutico. 
Quanto às principais etiologias relacionadas às disfunçôes sexuais femininas apresentadas neste serviço, referimos em ordem de freqüência: Conflitos e insatisfações conjugais, Falta de carinho, Falta de jogos sexuais, Desconhecimento do próprio corpo por formação repressiva ou crenças errôneas, Impedimento psicológico quanto ao direito ao prazer, Dinâmicas intrapsíquicas infantis comprometidas, Experiências sexuais violentas, Dificuldades com a menopausa, Problemas ginecológicos.

Como referimos, em alguns casos a queixa da mulher envolve seu parceiro e, para tal disponibilizamos neste ambulatório, o atendimento a homens. Quanto às disfunçōes masculinas diagnosticadas nestes serviço observamos: Disfunção Erétil (26.5\%), Ejaculação Precoce (24.5\%), Disfunção Erétil associada à Ejaculação Precoce (49\%). Em alguns casos, observamos que a Disfunção Erétil ou a Ejaculação Precoce apresentadas, também se encontravam associadas a outros diagnósticos como (peyronie, pênis torto congênito ou pênis pequeno). Os problemas masculinos tendo origens físicas, psicológicas ou sociais e em vários casos, as causas mistas foram à constante percebida.

Por estarmos em um serviço médico ginecológico, por vezes a cliente vem ao ambulatório imaginando que será uma consulta, uma medicação específica e pronto. Tal percepção faz com que tenhamos uma taxa de não retorno das paciente à consulta, em torno de quase $40 \%$ dos casos. Argumentam que não podem faltar ao trabalho, ou não têm com quem deixar as crianças, ou mesmo não dispõem de dinheiro para passagens freqüentes, vieram porque precisam, mas pensavam ser semelhante aos exames ginecológicos.

Porém a taxa de abandono do tratamento tem permanecido em menos de $8 \%$ dos casos, apontando para o fato, de que quando podem se disponibilizar para o tratamento, apesar das dificuldades que têm para o comparecimento, se esforçam bastante para obterem melhora em suas funções sexuais. Em alguns casos, quando a melhora já é razoável, como (na transformação de anorgasmia primária para secundária), argumentam que irão parar de vir, pois as dificuldades de comparecimento regular estão muito grandes e pensam que o que já conseguiram já é muito bom.

A variação do número de atendimentos, para que consideremos melhora significativa ou alta é bastante variável, e esta variação encontra-se diretamente relacionada ao tipo de problema apresentado, o envolvimento da paciente com o tratamento e a colaboração do parceiro, sendo que em (70\%) dos casos de disfunçōes, a faixa média de número de atendimentos para estes resultados, tem sido de 14 a 20 encontros. 
Quanto à análise de resultados por tipo de disfunção apresentada, observamos que foi nos casos de Vaginismo, que obtivemos a menor incidência de não retorno. Acreditamos que isso se deva ao real impedimento das atividades sexuais, o que implica em problemas para ambos do casal. Porém, no contraponto desta questão, quando as queixas delas, são associadas a problemas disfuncionais do companheiro, estes foram os casos de maior incidência de não retorno (quando o parceiro se negou ou não pode a vir).

Os casos de Transtorno de Desejo e Anorgasmia Secundária, associados a problemas conjugais como (alcoolismo, agressividade, infidelidade e desinteresse afetivo sexual), foram os responsáveis por nossos piores resultados. Porém nos casos em que o casal comparece regularmente aos atendimentos, possibilitando um trabalho conjuntos às dinâmicas conjugais e suas interferências na sexualidade, obtivemos bons resultados, apontando melhora ou alta em pouco mais da metade dos casos.

A transformação da anorgasmia primária em secundária é bastante freqüente (70\%). Porém a possibilidade de orgasmo à penetração bem menos freqüente. Principalmente quando associada à falta de carinhos e jogos sexuais preliminares à penetração. Queixa extremamente freqüente e persistente.

Quanto aos casos de disfunções masculinas, observamos bons resultados, especialmente vinculados a possibilidade de trabalho multidisciplinar que este ambulatório pode proporcionar. Onde avaliações e tratamentos médicos e psicológicos se complementam, propiciando melhores resultados.

Nos casos de má formaçōes genitais, como: Intersexo, Testículo feminilizante, ou Agenesia de Vagina, que requerem sempre atendimentos multidisciplinares, envolvendo dependendo do caso: terapia de reposição hormonal, cirurgia, exercícios, apoio psicológico, atendimento à família e incentivo constante. Excelentes resultados funcionais e de adaptação psicológica ao problema, têm sido obtidos.

Quanto ao Transtorno de Gênero, o acompanhamento exigido pelo Conselho de Medicina, é por mais de dois anos, onde atendimentos multidisciplinares, diagnóstico diferencial apurado, apoio psicológico constante, terapia de reposição hormonal, atendimento ao casal quando existente, dinâmica de grupo, esclarecimentos e reflexões quanto aos procedimentos cirúrgicos, cirurgia, apoio e tratamento no pós cirúrgico imediato e tardio, são as fases de tratamento. Quando todas as dificuldades inerentes ao sistema oficial de pagamento do SUS (Sistema Único de Saúde), assim como as dificuldades materiais do Hospital nos permitiram chegar em alguns poucos casos, a 
última etapa do processo, obtivemos excelentes resultados. Resultados estes, claramente obtidos pela dedicação e trabalho conjunto da equipe vinculada a este projeto.

\section{Referências bibliográficas}

ANDRADE-SILVA, M.C; CANELLA, P.R.B.; ROMERO, A.D.; BRUNO, R.E. Transtorno de gênero e Transgenitalização. Revista Brasileira de Sexualidade Humana. 16(2): 337-351, São Paulo: Iglu, 2005. BARBACH, L.J. For yourself, the fulfillment of female sexuality. New York: The New American Library, Inc, 1975.

BOKUS, F. Terapia do casal. Rio de Janeiro: Imago, 1980.

CANELLA, P.R.B.; ANDRADE-SILVA, M.C. Projeto de Gênero e Transgenitalização - Apresentação de dois casos clínicos. Revista Brasileira de Sexualidade Humana. 14 (2): 267-288. São Paulo: Iglu, 2003.

FIORINI, H. Teoria a Técnica de Psicoterapias. Rio de Janeiro: Francisco Alves., 1978

FIORINI. H. J.; PEYRÚ, G.D. Desenvolvimento em Psicoterapias. Rio de Janeiro: Francisco Alves, 1977.

HEIMAN, J. LO PICCOLO J.; LO PICCOLO, L. Descobrindo o prazer. São Paulo: Summus Editorial, 1981.

KAPLAN, H. S. The evaluation of sexual disorders: Psychological and Medical Aspects. New York: Brunner Mazel Publishers, 1983.

A nova terapia do sexo. Rio de Janeiro: Nova Fronteira, vol. 2., 1977.

A Nova Terapia do Sexo vol.2. O Desejo Sexual Rio de Janeiro:

Nova Fronteira, 1983.

Transtornos do desejo sexual. Porto Alegre: Artimed., 1999.

LAZARUS, A.A.Terapia Multimodal do Compartamento. São Paulo: Manole, 1977.

. Psicoterapia Personalista. Belo Horizonte: Interlivros, 1977.

MASTERS, H. W.; JOHNSON, V. E. Conduta sexual humana. Rio de Janeiro: Civilização Brasileira, 1966

Incompetência sexual - suas causas e tratamentos. Rio de Janeiro: Civilização Brasileira, 1970.

MASTERS, W. JOHONSON, V.; KOLODNY, R. Heterossexualidade. Rio de Janeiro: Bertrand Brasil, 1997.

PAPP, P. Casais em Perigo. Novas Diretrizes para Terapeutas Novas diretrizes para Terapeutas. In: PEGGY, P. (Org.). Porto Alegre: Artmed., 2002. ZILBERGELD, B. Male sexuality. New York: Bantam Books, 1978.. 